\title{
Role of Radio Frequency and Microwaves in Magnetic Fusion Plasma Research
}

\author{
(Invited Paper) \\ Hyeon K. Park ${ }^{1,2 *}$
}

\begin{abstract}
The role of electromagnetic (EM) waves in magnetic fusion plasma-ranging from radio frequency (RF) to microwaves—has been extremely important, and understanding of EM wave propagation and related technology in this field has significantly advanced magnetic fusion plasma research. Auxiliary heating and current drive systems, aided by various forms of high-power RF and microwave sources, have contributed to achieving the required steady-state operation of plasmas with high temperatures (i.e., up to approximately $10 \mathrm{keV}$; 1 $\mathrm{eV}=10000 \mathrm{~K})$ that are suitable for future fusion reactors. Here, various resonance values and cut-off characteristics of wave propagation in plasmas with a nonuniform magnetic field are used to optimize the efficiency of heating and current drive systems. In diagnostic applications, passive emissions and active sources in this frequency range are used to measure plasma parameters and dynamics; in particular, measurements of electron cyclotron emissions (ECEs) provide profile information regarding electron temperature. Recent developments in state-of-the-art 2D microwave imaging systems that measure fluctuations in electron temperature and density are largely based on ECE. The scattering process, phase delays, reflection/diffraction, and the polarization of actively launched EM waves provide us with the physics of magnetohydrodynamic instabilities and transport physics.
\end{abstract}

Key Words: Magnetic Fusion Plasmas, Plasma RF Heating, Plasma Diagnostics.

\section{INTRODUCTION}

In spite of fusion energy research having a long history, the cumulative results of fusion plasma research have converged to just one concept-namely, the magnetic fusion energy device. The apex of magnetic fusion energy research was the demonstration, almost three decades ago, of the scientific break-even (Q 1: ratio of output to input power) achieved in three large tokamak devices. Note that the devices were based on pulsed magnetic fields, and hence the steady-state capability could not be challenged. Following a long and intermittent period after this demonstration, fusion energy research has accelerated to its final stage. Technical and scientific progress has allowed re- searchers to challenge the electrical break-even (Q 10), through the International Thermonuclear Experimental Reactor (ITER), the first full-scale international science project; it is funded by a consortium of seven international partners (i.e., the European Union [EU], Japan, the United States, Russia, India, China, and South Korea). ITER success certainly warrants further development of a prototype fusion reactor (DEMO). The ITER device, based on superconducting magnets, will provide an opportunity to test the practical feasibility of fusion plasmas. The construction of the ITER is under way in southern France, and the first plasma is expected to take place around 2025. The pinnacle of ITER research will be a full deuterium-tritium (DT) experiment for $\mathrm{Q}$ 10, scheduled for around 2035. Among the many

Manuscript received September 29, 2017 ; Accepted October 17, 2017. (ID No. 20170929-057J)

${ }^{1}$ National Fusion Research Institute, Daejeon, Korea.

${ }^{2}$ Ulsan National Institute of Science and Technology, Ulsan, Korea.

"Corresponding Author: Hyeon K. Park (e-mail: hyeonpark@unist.ac.kr)

This is an Open-Access article distributed under the terms of the Creative Commons Attribution Non-Commercial License (http://creativecommons.org/licenses/by-nc/4.0) which permits unrestricted non-commercial use, distribution, and reproduction in any medium, provided the original work is properly cited.

(c) Copyright The Korean Institute of Electromagnetic Engineering and Science. All Rights Reserved. 
advances in fusion science and technology that are expected to derive from the ITER project, there are two critical results essential for DEMO. The first is the validation of $\alpha$-particle heating physics for self-sustained plasmas; the second is the quantification of a tritium breeding system for steady-state tritium fueling. After the goal of $Q=10$ is achieved in the ITER through the validation of self-sustainment in core heating and tritium fueling, the next step will be to demonstrate the production of electricity. Note that the long temporal gap that occurred between the two points (i.e., scientific and electrical break-even) did not occur for scientific reasons, but for political ones. To bridge the knowledge base derived from conventional tokamaks and that from the ITER device, new experimental tokamak devices based on a superconducting magnet and capable of steady-state operation have been successfully developed; the research focus with these devices will be the development of the science and technology essential to DEMO devices beyond the ITER.

Among the critical elements of fusion plasma research, the contribution of electromagnetic (EM) waves-from radio frequencies (RFs) to microwaves-has been significant. Here, an understanding of the propagation characteristics of EM waves in plasmas has been critical to efficient auxiliary heating and external current drive, as well as to diagnostic applications. The wave physics associated with the penetration of the evanescent region in the plasma edges and propagation to the core plasma are extremely rich and complex in magnetically confined plasmas, where the magnetic field and dielectric property are not uniform. A high-power RF source is employed to heat the ions through resonance like that seen in an ion-ion hybrid, or second harmonic resonances [1, 2]. High-power microwave sources (e.g., a gyrotron) have been used to heat the electrons via electron cyclotron resonance $[3,4]$; the heating/current drive scheme based on these sources is relatively straightforward, since there is no evanescent region between the launcher and resonance layers, and a sufficiently high frequency can preclude cutoffs at high-density plasmas.
The diagnostic application of EM waves [5] for high-temperature plasmas confined to a magnetic field requires a full understanding of wave interaction with the plasma, whether it is passively emitted from the plasmas or actively launched to the plasma. One example of passive emission is electron cyclotron emission (ECE) in radiometry [5]; ECEs in fundamental and second harmonics have been used for electron temperature profile measurement. In recent years, owing to advances in microwave technology and data processing, 2D/3D imaging systems [6] that leverage ECE have been developed to generate new and detailed physics information regarding magnetohydrodynamic (MHD) instabilities and turbulence. In active diagnostics [5], both reflectometry (for electron density profiles and their fluctuations) and collective scattering systems have also provided momentum-resolved electron density fluctuations in Fourier (wave number and frequency) space. The effects of phase delay and Faraday rotation are used to measure the line-integrated electron density and poloidal magnetic field that is critical to plasma stability and confinement.

\section{PROGRESS IN MAGNETIC FuSION RESEARCH}

\section{AND KSTAR}

The history of fusion plasma research over more than one half-century has seen a variety of concepts and devices. That long history finally converged to the tokamak configuration (i.e., fusion plasma confined to a toroidal and poloidal magnetic field), which has been proven to have the most stable plasmas with adequate energy confinement time. The tokamak concept, conceived by Soviet physicists Tamm and Sakharov in the 1950s, finally led to experiments in which the "break-even" was challenged, in three large tokamak devices-namely, the Tokamak Fusion Test Reactor (TFTR) (United States) [7], the Joint European Tokamak (JET), (the EU) [8], and the Japan Tokamak 60 Upgrade (JT-60U) (Japan) [9]) (Fig. 1). Here, the "breakeven" implies that $Q=1$, where $Q$ is the ratio of output energy to input energy. Based on advances in technology and science

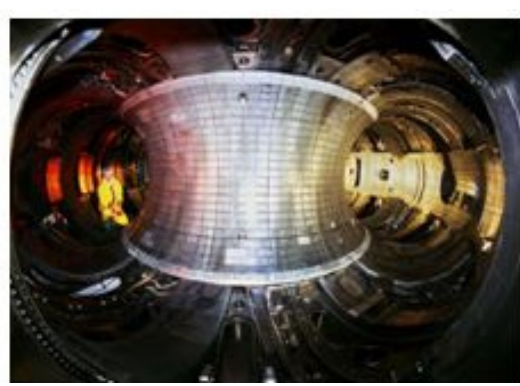

(a)

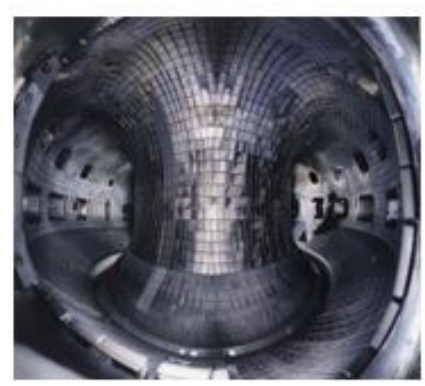

(b)

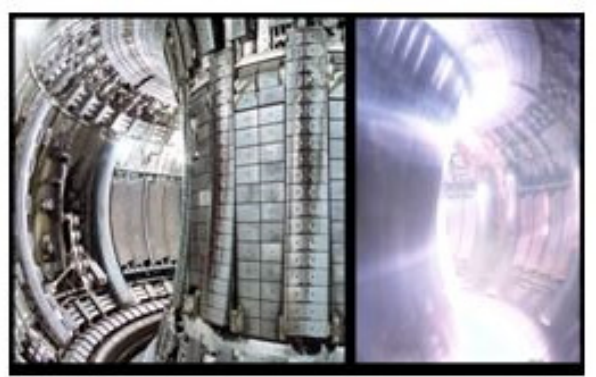

(c)

Fig. 1. Fusion power production experiments close to Q 1 with DT fueling (DD equivalent) were achieved with three large tokamak devices (TFTR and JET experiments were based on DT fueling, and the JT-60U experiment was a projection based on DD fueling).

(a) Internal view of TFTR, (b) internal view of JT-60U, (c) internal view with and without plasma in JET. 
grounded in these successful experiments, fusion plasma research has reached a point where the "electrical break-even" (Q 10) can be challenged through the construction of the ITER.

The ITER project, which has been discussed for more than three decades, finally materialized as the first large-scale international science project. It is jointly financed by the seven aforementioned economic regions, and its construction is proceeding "full steam ahead" in southern France. As mentioned, it is projected that the ITER device will be operational by around 2025, and the ultimate goal of $Q=10$ (i.e., approximately $500 \mathrm{MW}$ of fusion power) with DT fuel is scheduled to occur around 2035. It is important to be aware of two critical elements of the ITER project for fusion energy development beyond the ITER era, neither of which can be achieved with current-generation experimental devices. The first is the validation and quantification of the physics of " $\alpha$-particle heating," in which sufficient reheating of the burn products from a DT reaction $[D+T=\mathrm{n}(1.6 \mathrm{MeV})$ $\left.+\mathrm{He}_{3}(3.45 \mathrm{MeV})\right]$ can sustain the burning plasmas. The second important technical issue is the quantification of tritium fuel production from the tritium blanket module, so that selfbreeding of the fueling can be demonstrated in the ITER. Successful achievement of Q 10 with a clear and successful assessment of these two critical ITER experimental results will herald the dawn of viable fusion energy generation. From there, the race for electricity production by fusion reactor will begin, perhaps in those countries participating in the ITER project.

Following the fruitful demonstration of $\mathrm{Q} \sim 1$ experiments in three large tokamaks based on devices with magnets made of copper-which limit the pulse length to less than approximately 10 seconds-the fusion community has envisaged the development of a steady-state-capable tokamak device. Following many attempts and proposals, new-generation tokamaks based on superconducting coils have been developed, and they challenge the steady-state operation of tokamak devices. One of the leading devices is the Korean Superconducting Tokamak Advanced Research (KSTAR) [10, 11], commissioned in 2008. The KSTAR was constructed by South Korean engineers and industries as a fully superconducting tokamak device, and its design derived from collaboration between the United States and South Korean scientists. The poloidal cross-section with and without plasma, as well as a schematic of internal structures, are illustrated in Fig. 2. KSTAR research has made significant progress in terms of tokamak plasma science and technology, toward steady-state operation. The KSTAR has proven to be an excellent fusion plasma research facility, and it is equipped with three unique features. First, the KSTAR is the most precisely engineered tokamak device anywhere, with the lowest error field and lowest magnetic ripple of any tokamak device ever built

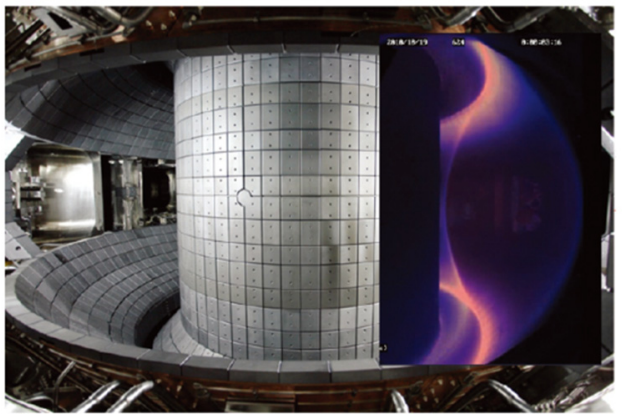

(a)

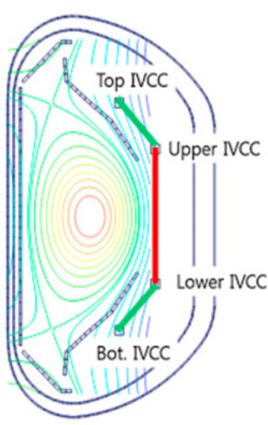

(b)
Fig. 2. (a) Internal view of the KSTAR device, where the inner wall and divertor are shown with and without the plasma. (b) The poloidal cross-section view of the internal control systems; the IVCC coils and passive plate are illustrated.

[12]. (It is well known that the stability and confinement of the plasmas in magnetic confinement devices are affected by the helical asymmetry of the magnetic field, which arises from the error field and magnetic ripples.) Hence, the KSTAR is an excellent test bed for studying the influence of helical asymmetry on stability and confinement in tokamak plasmas. Second, the KSTAR is equipped with state-of-the-art in-vessel control coils (IVCCs) with low toroidal mode numbers $(\mathrm{n}=1$ and $\mathrm{n}=2)$ [11]; thus, the IVCCs can be used as a magnetic perturbation tool to test the effect of asymmetries, and to control harmful MHDs. This combination of an almost-perfect symmetric magnetic field and perturbation tool allows for the systematic testing of stability and confinement physics. The last unique feature of the KSTAR is its state-of-the-art 2D microwave imaging system-which employs microwave imaging reflectometry (MIR) and a 2D/3D electron cyclotron emission imaging (ECEI) system-which can visualize the MHD and turbulence physics at excellent spatial and temporal resolutions $[13,14]$. These diagnostic systems have demonstrated new physics not available in the past with $1 \mathrm{D}$ diagnostic systems. These advanced diagnostic systems are instrumental to the study of the unresolved physics of MHDs and turbulence-related transport.

There are currently two goals driving KSTAR research. The first goal is to verify the science and technology essential to ITER and K-DEMO program. Here, the focus is on developing an operation regime that can minimize harmful MHD instabilities and a divertor system that can remove the heat transported from the plasma to the divertor. The second goal is to develop the human resources essential to the South Korean fusion energy development plan, which consists of the KSTAR project, partnership in ITER, and the Korean DEMO program. Note that South Korea is responsible for approximately 100 scientists for the ITER program, and many more engineers for the K-DEMO program. 


\section{APPLICATION OF RADIO FREQUENCIES AND}

\section{MiCROWAVES IN TOKAMAK PLASMAS}

The principle of the tokamak plasma device is based on the transformer, as the plasma is the secondary current and the primary current is driven by $\mathrm{Ohmic}$ heating coils. The temperature (ions and electrons) of the plasma is initially increased through the Ohmic heating process by the induced secondary current in the plasma. Note that the poloidal field generated by the plasma current is critical to sustaining plasma equilibrium. The electron temperature increased by the Ohmic current is slowed and saturated at approximately 1-2 keV, as the efficiency of the Ohmic heating is diminished due to reduced resistivity as the plasma temperature is increased. To increase the temperature that is optimal for fusion reactivity (approximately $10 \mathrm{keV}$ ) for the DT fusion reactor, external heating needs to be provided. Note that core heating will be provided by $\alpha$-particle $\left(\mathrm{He}_{3}\right)$, a by-product of DT reactions in the DT-based fusion reactor. At the same time, for steady-state operation, the diminished plasma current due to reduced Ohmic current efficiency also needs to be compensated by external means, even though the large portion of the current is provided by self-generated current known as bootstrap current.

In heating and current drive schemes in a tokamak device, those methods that make use of resonance characteristics (e.g., cyclotron resonance for ions and electrons) have been attractive. Since there are two main species in the plasma (i.e., ions and electrons) and two resonance frequencies differ by inverse of the square root of the mass (i.e., high frequency for electrons and low frequency for ions). For the strength of magnetic fields in current research devices, the frequency ranges from $\mathrm{RF}$ to $\mathrm{mi}-$ crowave. The ion cyclotron frequency falls within the RF range (approximately $40-500 \mathrm{MHz}$ ) and the electron cyclotron frequency falls within the microwave range (approximately 50$300 \mathrm{GHz}$ ). For a heating and current drive to be effective, the required power may be up to $10 \mathrm{MW}$. The critical issue that arises with a heating and current drive that uses EM waves in the plasma is the coupling problem. A high frequency exceeding approximately $50 \mathrm{GHz}$ is relatively easy to achieve, since there is no evanescent region from the launcher to the plasma and the power can be delivered directly to the resonance layer. However, one needs to be conscious of the cut-off layer at a high edge density, which can be found in an $\mathrm{H}$-mode operation in tokamak plasmas. The most commonly known high-frequency source is gyrotrons, with the output power of one unit being up to approximately $1 \mathrm{MW}$; the transmission line consists of lowloss corrugated waveguides. On the other hand, for an effective heating scheme, the coupling of low-frequency RFs requires an effective antenna design that can couple the power to the plasmas through the evanescent layer at the edge of the plasma, by way of the wave propagation physics (dispersion property) of $\mathrm{RFs}$ in a non-uniform dielectric media (i.e., a nonuniform magnetic field and plasma pressure). The transmission line runs through an over-mode waveguide system to the antenna structure. In most devices, heating through the mode-converted ion Bernstein wave (IBW) at hybrid resonance or at the second harmonic resonance is employed to heat the plasmas. In devices with a low magnetic field $(<1 \mathrm{~T})$, higher harmonic fast waves are used to heat the plasma. Another scheme uses Helicon waves in a higher magnetic field, where the antenna needs to line up with the pitching magnetic field at the edge of the tokamak. An effective current drive is achieved through lower hybrid resonance with a frequency range of approximately 2-5 $\mathrm{GHz}$ and the directional launching of electron cyclotron resonance heating $(\mathrm{ECH})$ waves (i.e., plasma current direction or opposite direction).

The plasmas consist of charged particles (e.g., ions, impurities, and electrons) in a nonuniform magnetic field; there is a peak temperature ranging from a few $\mathrm{eV}$ to tens of $\mathrm{keV}$, and a peak electron density of approximately $1 \times 10^{19}$ to $1 \times 10^{20} \mathrm{~cm}^{-3}$. RFs and microwaves have been actively used to diagnose plasma parameters and associated dynamics. First, emissions from the charged particles (e.g., the main ion, impurity ions, and electrons) due to acceleration in an electric and magnetic field, as well as the ionization and recombination process, have been widely used to measure plasma parameters. As examples, ion temperature and plasma rotation can be measured using the broadened line width and shift of the emission line, by way of the Doppler effect (e.g., X-ray crystal spectroscopy and charge exchange recombination). The motional Stark effect of emissions from the energetic external beam ions has been used for magnetic field measurement. Additionally, the Bremsstrahlung emission-which is strongly dependent on electron temperature and densityis used to estimate both electron density and temperature. Emissions from the cyclotron motion of the ions and electrons in the $\mathrm{RF}$ and microwave range are extremely rich, and they have been used to study plasma dynamics and measure plasma parameters. Emissions in the RF range correspond to the motion of ions at a resonance and in wave propagation (e.g., whistler waves, Alfven waves, and in cyclotron harmonics). In particular, emissions at the microwave range correspond to the dynamics of electrons. RFs and microwaves have been actively used to diagnose these plasma parameters, such as the temperature and density of ions and electrons, and plasma current distribution. Here, the phase delay of transmitted or reflected waves is used to measure the plasma density, as part of an interferometer or reflectometry [15] system. The measurement of scattered EM waves [16] from coherent waves and turbulent plasma fluctuations is used to determine the dispersion of the local wave propagation, as well as fluctuating wave numbers and corresponding frequencies. An 
interferometer/polarimeter system [17] measures line-integrated density and magnetic fields (i.e., poloidal and toroidal), using the Faraday rotation effect. High-temperature plasmas confined to a magnetic field are also rich in emissions of a broad wavelength range, from RFs to gamma rays. The best example is ECE, which is in the range of $50-500 \mathrm{GHz}$. The novel character of ECE as a blackbody has been widely used to measure the local electron temperature, where the intensity of ECE is proportional to the electron temperature in the plasmas and the frequency corresponds to the local magnetic field [5]. The advancement of detection array technology and signal processing may enable the development of an imaging system used to visualize MHDs and turbulence [6].

\section{Examples of Heating and Current Drive in the Plasma}

Various RF forms (ranging from approximately 50 to 500 $\mathrm{MHz}$ ) and microwaves (ranging from approximately 2 to 100 $\mathrm{GHz}$ ) have been developed for heating and current drive in magnetically confined plasmas. Note that the in-plasma heating method is largely based on resonance between RF and plasma particles (e.g., ions and electrons), with wave energy being effectively converted into particle energy. For ion cyclotron heating (ICH) at a frequency ranging from approximately 50 to 500 $\mathrm{MHz}$, once the resonance characteristics in the machine (e.g., the second harmonic ion cyclotron or the hybrid resonance layers in the plasma) are identified, one can design a transmission line and an appropriate launching antenna to be located at the edge of the plasmas. Then, the study of the coupling of RF power to the edge plasmas through the evanescent layer can be modeled to optimize the power coupling to the plasma. The coupled RF power at the edge of the plasma needs to propagate in a fast-wave form until it reaches the resonance layers [18]; then, the fast wave is converted into electrostatic waves (such as the IBW) to transfer the wave energy to the particles. The modeling for overall wave propagation has been developed so as to understand the heating process while using a full-wave solution. An ICH process based on a mode-converted IBW is a good example. Here, the long-wavelength fast wave launched from the lower field side encounters the hybrid resonance in the core of the plasma. The 3D modeling results are achieved using a full-wave modeling tool (N. Bertelli, personal communication Dec. 19, 2016) (Fig. 3). The ion heating with RF is not limited to the second ion cyclotron harmonic or hybrid resonance frequencies. The application of higher harmonic frequencies has been successfully demonstrated in a device with a lower magnetic field, such as NSTX [18]. Even higher-RF frequencies (approximately $500 \mathrm{MHz}$ ), as a form of helicon waves, is challenged in KSTAR [19] and DIII-D. While the ICH approximates the ion cyclotron frequency, the RF energy directly heats the ions, and the ions are immediately slowed down by the
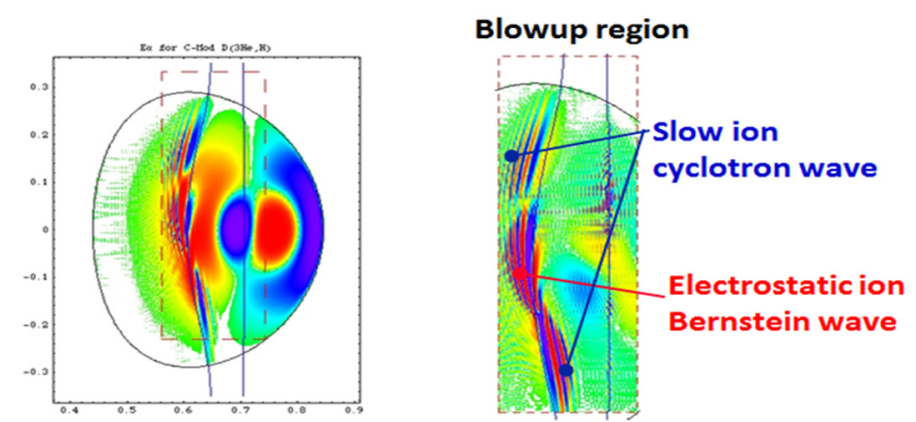

Fig. 3. Propagation of the long-wavelength EM waves (fast-wave branch) from the low field side and converted into electrostatic waves (ion Bernstein waves) at the hybrid resonance layer in the core of the plasma (N. Bertelli, personal communication, Dec. 19, 2016).

electrons. As a result, the electron temperature is significantly increased. Other issues encountered in practice are antenna complexity and the long conditioning time needed to optimize the wave power coupling.

For electron heating in the plasma, $\mathrm{ECH}$ has been most popular. Here, the fundamental and second harmonic electron resonance frequencies that fall within the microwave range (approximately 50-300 GHz) for magnetic fields ranging from approximately $1 \mathrm{~T}$ to $5 \mathrm{~T}$ are used. Here, high-power gyrotrons are used to deliver the power to the plasmas through a low-loss waveguide and a quasi-optical launcher attached to the transmitting waveguide. Generally, the launcher can steer the beam to where the heating and current drive can be carried out effectively. As far as electron heating is concerned, ECH is very effective and coupling is simple, since the launcher system requires only a small area relative to the large antenna area required for RF heating.

As mentioned, the external current drive is essential for the steady-state operation of the tokamak plasma, in which the equilibrium is sustained with the poloidal magnetic field induced by the current in the toroidal direction. For an effective external current drive for the steady-state operation of tokamak plasmas, RFs are extensively used to transfer the momentum of ions or electrons. The most effective methods are based on lower hybrid resonance frequencies (approximately $2-5 \mathrm{GHz}$ ), fastwave propagation at higher harmonic frequencies $\sim 50$ $500 \mathrm{MHz}$ ), and the electron cyclotron frequency (approximately 80-200 GHz). The key research issues include the development of stable and robust high-power RF (up to approximately $500 \mathrm{MHz}$ ), klystrons (up to approximately $5 \mathrm{GHz}$ ), and gyrotrons (approximately 50-200 GHz), and the design and fabrication of robust launchers for RF and microwaves. Most importantly, it is necessary to have a full understanding of the physics of wave propagation and the absorption/damping process in plasmas, aided by the modeling of wave propagation and 
coupling in the plasma. In particular, efficient penetration and propagation to the plasma at higher beta plasmas are key issues for all current drive systems.

\section{Examples of Microwave Diagnostics for Plasma Dynamics and Profiles}

The best example of emissions-based diagnostics is arguably an ECE system based on the fundamental and second harmonics of ECE, which is used to measure the local electron temperature and fluctuations thereof. Here, optical depth is important, and the emission can be treated as a blackbody radiation as long as the optical depth is thick (i.e., a reasonably high electron density). In an optically thin region, the emission no longer represents the local electron temperature. The measurement of electron temperature based on the conventional ECE system (Fig. 4(a)) has been extremely reliable. Here, plasma emissions at a wide frequency range $\left(\omega_{e c}=\frac{e B}{m_{e}}, B \propto \frac{1}{R}\right)$ are mixed with a sweeping local oscillator at a single detector; the intermediate frequency band is integrated for each position where $e$ and $m_{e}$ is the charge and mass of the electron, respective$1 y$, and $B$ is the local magnetic field that depends on the major radius $\mathrm{I}$ (as shown in this figure). With advances in microwave technology, it has become possible to develop a 2D microwave imaging system that can measure electron dynamics (such as MHD instabilities and temperature turbulence) at high spatial and temporal resolutions. A schematic of a 2D ECE imaging system employing a $1 \mathrm{D}$ array detector system is shown in Fig. 4(b); the $1 \mathrm{D}$ vertical array consists of 24 single detectors and has one-to-one mapping to the vertical position of the emission in the plasma. The wideband frequency (approximately $10 \mathrm{GHz}$ ) in the radial span is divided into frequency bins (approximately 700-900 MHz) and simultaneously processed to form a radial resolution. A total of 192 pixels $(24 \times 8)$ form an image of the electron motion.
The first 2D ECEI system [6] was tested on TEXTOR plasma, and was used to study the physics of a sawtooth crash (Fig. 5). Sawtooth instability is a repetitive oscillation (i.e., repetitive slow rise and sudden collapse of the core plasma pressure, as shown on the left-hand side of this figure) caused by the reconnection process of the $m=n=1 / 1$ kink instability in the core of tokamak plasma, where $m$ and $n$ are poloidal and toroidal mode numbers, respectively. The changes in electron temperature in $2 \mathrm{D}$ during the crash time are illustrated on the right-hand side of this figure. When the core pressure exceeds a critical value, the core pressure is transported to the mixing zone (outside of the inversion layer) through the reconnection process. The accumulated pressure in the mixing zone is transported in a diffusion time scale. Since the successful implementation of the ECEI system on the TEXTOR, ECEI systems have become standard diagnostic systems on most tokamak devices, including KSTAR.

Additionally, high-temperature plasma has a unique dielectric property, and the propagation characteristics of externally launched EM waves have been widely used to measure plasma properties. For active non-perturbing diagnostic applications, the transmitted, reflected, and scattered part of the externally launched EM waves has been used to probe the macroscopic instabilities and underlying physics of energy transport, in addition to measuring plasma parameters. These comprise coherent and incoherent Thomson scattering, for electron temperature, density, and fluctuations; interferometry and polarimetry, for electron density and magnetic field measurement; and reflectometry, for electron density and fluctuations. The first example can be reflectometry based on the reflection position of the externally launched microwave at the cut-off layer. Here, the externally launched wave approaches the cut-off layer, the wavelength approaches infinity, and the electric field swells. The launched frequency corresponds to the electron density of the reflection position. Additionally, the frequency broadening of

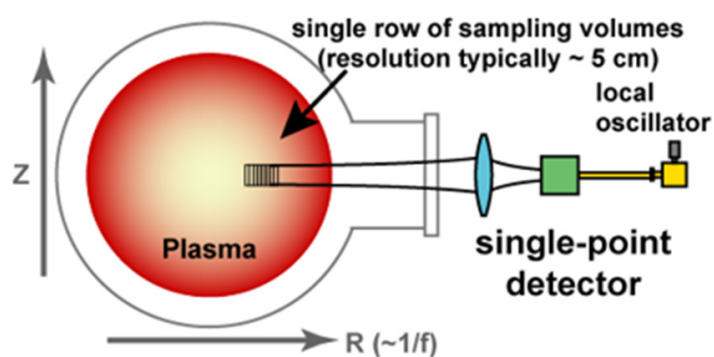

Conventional 1-D ECE system

(a)

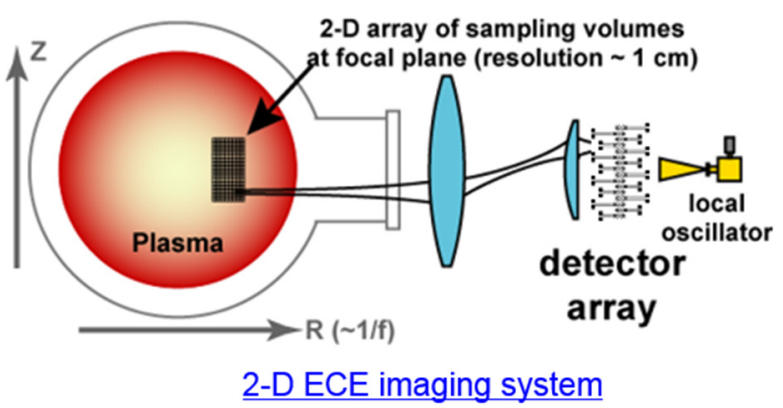

(b)

Fig. 4. Schematics of a conventional ECE system and an ECEI system. (a) In a conventional system, emissions from the plasma on the mid-plane are collected with a single detector and the frequency is down-converted with the sweeping local oscillator source. (b) Large optics are used to collect 2D emissions from the plasma. Vertical coverage is mapped to the 1D array detector (24), and each detector performs like a single-point detector. 

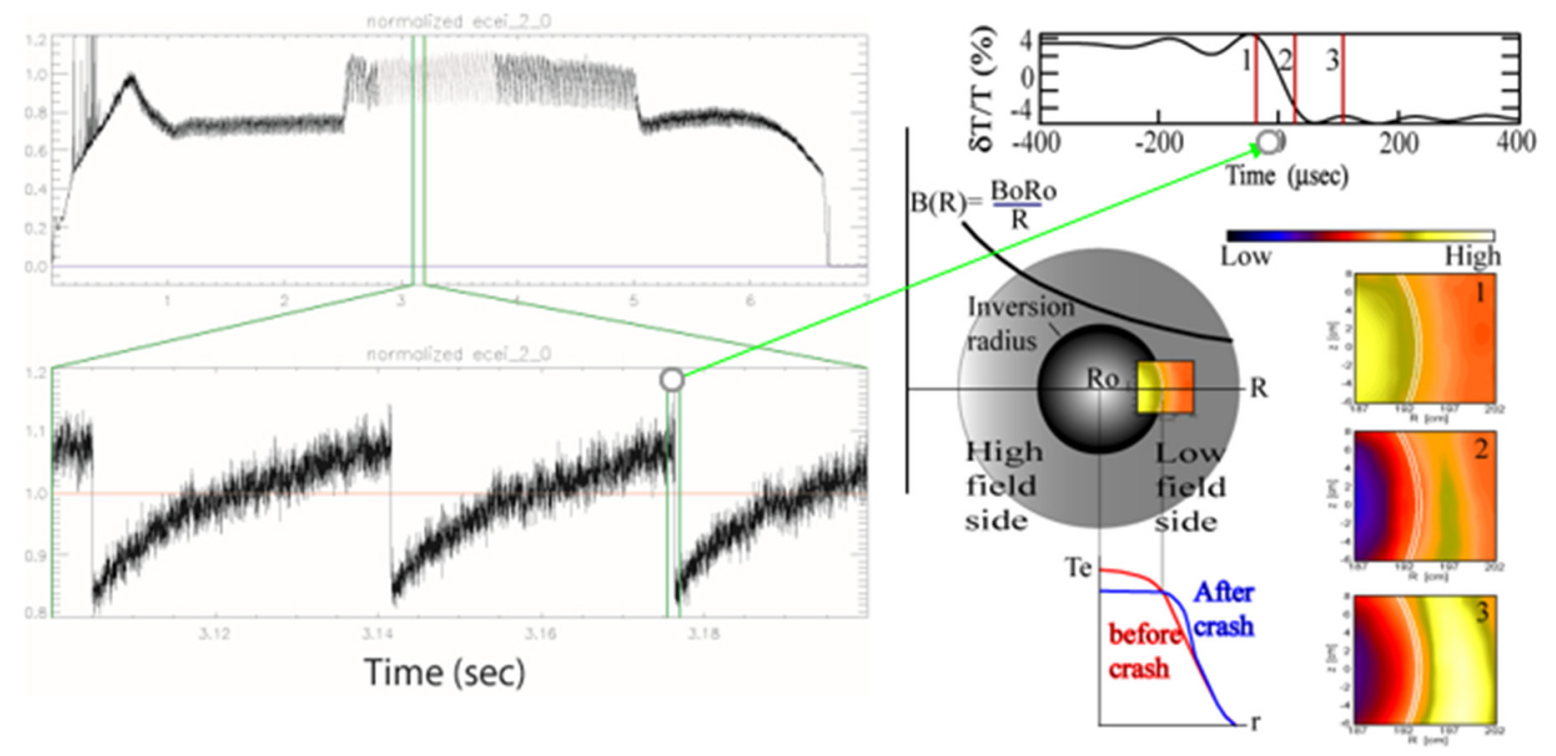

Fig. 5. The 2D images measured by ECEI system are illustrated in color. Increased core electron temperature is represented by yellow color before the crash (1) and increased temperature outside of the inversion radius (white line) from the core is trapped in the mixing zone (3). The fast change in electron temperature during the reconnection process is progressing in (2).

the reflected waves (due to the turbulent motion of the electrons along the propagation direction to the cut-off layer) contains weighted information on the electron turbulence of the cut-off layer. The second example can be a scattering of an EM wave from the collective motion of the electrons in the plasma. Here, one can measure fluctuations in Fourier space $(\omega, k)$, based on momentum matching $\left(k_{s}=k_{0}-k_{w}\right.$; Bragg condition) and energy conservation $\left(\omega_{s}=\omega_{0}-\omega_{w}\right)$, where the subscript $s$ denotes scattered waves, $O$ denotes the probe beam, and $w$ denotes plasma waves. The third example is interferometry/polarimetry for electron density and magnetic field measurement. When the linearly polarized wave propagates through the plasma in a magnetic field, the transmitted wave experiences phase delay, compared to the same beam path without plasma. The polarization direction is changed due to the difference in propagation speed between the right-hand and left-hand polarized waves (i.e., Faraday rotation effect). Note that the linearly polarized wave is the sum of these two circularly polarized waves. The phase delay of the transmitted waves with respect to the waves without plasma is proportional to the line-integrated electron density along the propagation path, and polarization rotation is proportional to the line-integral value of the product of the magnetic field along the propagation direction and the electron density. Frequently, the chordal measurement of the plasma has been used to measure both plasma density and the magnetic field.

\section{SUMMARY}

A wide range of EM waves-from RF to gamma rays-serve as essential elements in fusion plasma physics research. In particular, high-power sources (i.e., those exceeding the multi-MW level), ranging from RFs to microwaves, have been widely used to heat plasma, achieve plasma temperatures up to approximately $10 \mathrm{keV}$, and achieve steady-state operations. Here, the resonance characteristics of ions, electrons, and impurities are used to transfer EM wave energy; additionally, hybrid resonance is introduced in the core of the plasma to convert EM waves to electrostatic waves-such as ion Bernstein waves-to transfer RF energy to particles in the plasmas. For diagnostic applications, there are two aspects to consider. The first is the use of passive measurements of natural emissions in the RF and microwave range frequency to measure plasma parameters and related dynamics. The example given here was electron temperature profile measurement by ECE. Based on the robust measurement of electron temperatures via $\mathrm{ECE}$, a $2 \mathrm{D}$ imaging system for electron fluctuation measurement was developed; it reveals many new aspects of MHD physics that are not available through the use of conventional diagnostic systems. The second is the active system used to detect reflected waves used to assess the electron density profile and fluctuation, and scattered waves in Fourier space to study momentum-resolved electron density fluctuations. By measuring the phase delay and the change in polarization of the transmitted waves, the line-integrated density and magnetic field can be measured.

This work is supported by the National Research Foundation of Korea (NRF) grant (No. NRF-2014M1A7A1A03029865). 


\section{REFERENCES}

[1] T. H. Stix, Waves in Plasmas. New York, NY: AIP Press, 1992.

[2] J. Adam, "Review of tokamak plasma heating by wave damping in the ion cyclotron range of frequency," Plasma Physics and Controlled Fusion, vol. 29, no. 4, pp. 443-472, 1987.

[3] A. C. Riviere, "A review of ECRH experiments," Plasma Physics and Controlled Fusion, vol. 28, no. 9A, pp. 1263-1276, 1986.

[4] M. R. de Baar, M. N. A. Beurskens, G. M. D. Hogeweij, and N. J. Lopes Cardozo, "Tokamak plasmas with dominant electron cyclotron heating; evidence for electron thermal transport barriers," Physics of Plasma, vol. 6, no. 12, pp. 46454657, 1999.

[5] I. H. Hutchinson, Principles of Plasma Diagnostics. New York, NY: Cambridge University Press, 2009.

[6] H. Park, E. Mazzucato, T. Munsat, C. W. Domier, M. Johnson, N. C. Luhmann et al., "Simultaneous microwave imaging system for density and temperature fluctuation measurements on TEXTOR (invited)," Review of Scientific Instruments, vol. 75, no. 10, pp. 3787-3792, 2004.

[7] R. J. Hawryluk, "Review of D-T experiments relevant to burning plasma issues," Journal of Plasma and Fusion Research Series, vol. 5, pp. 12-21, 2002.

[8] J. Jacquinot, V. P. Bhatnagar, J. G. Cordey, L. D. Horton, D. F. H. Start, R. Barnsley et al., "Overview of ITER physics deuterium-tritium experiments in JET," Nuclear Fusion, vol. 39, no. 2, pp. 235-254, 1999.

[9] K. Tobita, "Latest plasma performance and experiments on JT-60U," Plasma Physics and Controlled Fusion, vol. 41, no. 3A, pp. 333-344, 1999.

[10] M. Kwon, Y. K. Oh, H. L. Yang, H. K. Na, Y. S. Kim, J. G. Kwak et al., "Overview of KSTAR initial operation," $\mathrm{Nu}$ clear Fusion, vol. 51, no. 9, article no. 094006, 2011.

[11] J. G. Kwak, Y. K. Oh, H. L. Yang, K. R. Park, Y. S. Kim, W. C. Kim et al., "An overview of KSTAR results," Nuclear
Fusion, vol. 53, no. 10, article no. 104005, 2013.

[12] Y. In, J. K. Park, J. M. Jeon, J. Kim, and M. Okabayashi, "Extremely low intrinsic non-axisymmetric field in KSTAR and its implications," Nuclear Fusion, vol. 55, no. 4, article no. 043004, 2015.

[13] G. S. Yun, W. Lee, M. J. Choi, J. B. Kim, H. K. Park, C. W. Domier et al., "Development of KSTAR ECE imaging system for measurement of temperature fluctuations and edge density fluctuations," Review of Scientific Instruments, vol. 81, no. 10, article no. 10D930, 2010.

[14] W. Lee, J. Leem, J. A. Lee, Y. B. Nam, M. Kim, G. S. Yun et al., "Microwave imaging reflectometry for density fluctuation measurement on KSTAR," Nuclear Fusion, vol. 54, no. 2, article no. 023012, 2014.

[15] R. Nazikian, G. J. Kramer, and E. Valeo, "A tutorial on the basic principles of microwave reflectometry applied to fluctuation measurements in fusion plasmas," Physics of Plasmas, vol. 8, no. 5, pp. 1840-1855, 2001.

[16] D. R. Smith, E. Mazzucato, T. Munsat, H. Park, D. Johnson, L. Lin, C. W. Domier, M. Johnson, and N. C. Luhmann, "Microwave scattering system design for electron scale turbulence measurements on NSTX," Review of Scientific Instruments, vol. 75, no. 10, pp. 3840-3842, 2004.

[17] H. K. Park, C. W. Domier, W. R. Geck, and N. C. Luhmann, "Far infrared tangential interferometry/polarimetry on the National Spherical Tokamak Experiment," Review of Scientific Instruments, vol. 70, no. 1, pp. 710-713, 1999.

[18] N. Bertelli, E. F. Jaeger, J. C. Hosea, C. K. Phillips, L. Berry, P. T. Bonoli et al., "Full wave simulations of fast wave efficiency and power losses in the scrape-off layer of tokamak plasmas in mid/high harmonic and minority heating regimes," Nuclear Fusion, vol. 56, no. 1, article no. 016019, 2016.

[19] S. J. Wang, J. Kim, J. H. Jeong, H. J. Kim, M. Joung, Y. S. Bae, and J. G Kwak, "Recent experimental results of KSTAR RF heating and current drive," AIP Conference Proceedings, vol. 1689, no. 1, article no. 030014, 2015. 
Hyeon K. Park

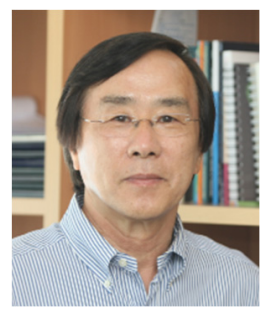

received a B.S. in physics, USC (1978), and M.S. and $\mathrm{Ph} . \mathrm{D}$. degrees in electrical engineering, UCLA (1980 and 1984). His research was on pioneering diagnostic development in the $\mathrm{THz}$ range. He started his career as a member of the research staff at Princeton Plasma Physics Laboratory, Princeton University, where he worked until late 2007; his final rank there was Principal Research Physicist. He worked on the TFTR device, which was the flagship object of US fusion research. Later, he successfully developed the world's first microwave imaging diagnostics, in the TEXTOR device in Germany. In late 2007, he was appointed as a professor of physics, POSTECH, South Korea, and in 2009 he established one of the fusion plasma research centers at POSTECH. There, he developed a state-of-the-art 2D microwave imaging system for KSTAR, which has been instrumental to KSTAR physics research. In 2013, he moved to the physics department at UNIST, South Korea, where he established a new fusion research center for plasma stability and transport. He has published approximately 300 SCI papers, including around 36 PRLs. He has delivered many plenary and invited talks at major international conferences, including those of AAPS-DPP, EPS-DPP, and APSDPP. He has served on many international committees, including the International Fusion Research Council and the ITER Science and Technology Advisory committee, and has been a Chair and Co-Chair of the ITPA Diagnostic Division. He has also served as an editorial board member for Plasma Physics and Controlled Fusion, and he is a Fellow of American Physical Society. 